
allemande

45-2 | 2013

Images et discours de la nation

\title{
Emblème interactif du commerce identitaire : « Bitte liebt Österreich » de Christoph Schlingensief
}

Kerstin Hausbei

\section{(2) OpenEdition}

Journals

Édition électronique

URL : https://journals.openedition.org/allemagne/1484

DOI : 10.4000/allemagne. 1484

ISSN : 2605-7913

Éditeur

Société d'études allemandes

Édition imprimée

Date de publication : 30 décembre 2013

Pagination : 283-296

ISSN : 0035-0974

\section{Référence électronique}

Kerstin Hausbei, « Emblème interactif du commerce identitaire : «Bitte liebt Österreich » de Christoph Schlingensief », Revue d'Allemagne et des pays de langue allemande [En ligne], 45-2 | 2013, mis en ligne le 29 juillet 2019, consulté le 19 mai 2021. URL : http://journals.openedition.org/allemagne/1484 ;

DOI : https://doi.org/10.4000/allemagne.1484 


\section{Emblème interactif du commerce identitaire: « Bitte liebt Österreich » de Christoph Schlingensief}

- Kerstin Hausbei*

Dans le théâtre européen, les œuvres de commande ayant trait à l'«identité nationale » appartiennent à un passé révolu. Pourtant, une des œuvres les plus connues de Christoph Schlingensief, l'installation-performance (Aktion) « Bitte liebt Österreich. Erste österreichische Koalitionswoche » réalisée en juin 2000 dans le centre historique de Vienne, y ressemble de près.

En réplique à l'émission télévisée Big Brother ${ }^{(1)}$ dont le lancement en mars 2000 avait inauguré l'ère de la " télé-réalité » sur les chaînes de langue allemande et déclenché si ce n'est une "panique morale " du moins une "panique médiatique " ${ }^{(2)}$, Schlingensief avait installé douze demandeurs d'asile dans un "village de containers » ${ }^{(3)}$, des baraques de chantier où ils furent filmés en continu. L'installation était surmontée d'un écriteau portant le slogan " Ausländer raus » ("Étrangers dehors »), du logo du tabloïd autrichien Kronen Zeitung et du drapeau du FPÖ. Des affiches électorales du FPÖ et la retransmission de discours électoraux de Jörg Haider complétèrent le décor visuel et sonore. Comme dans l'émission télévisée, les candidats devaient se soumettre à certains rituels et jeux collectifs imposés. Via un vote par téléphone, ils furent éliminés à un rythme régulier

* Maître de conférences, Université Paris 3 Sorbonne Nouvelle, membre du CEREG.

1 Le format fut lancé par Endemol en octobre 1999 aux Pays-Bas sur la chaîne privée Veronica, puis repris dans d'autres pays, également sur des chaînes privées à faible audience, ainsi sur RTL2 pour les pays germanophones en mars 2000 et sur M6 (sous le titre Loft story) en France en avril 2001.

2 Cf. Daniel Biltereyst, «Reality TV, troublesome pictures and panics: reappraising the public controversy around Reality TV in Europe », in: Su Holmes et Deborah Jermyn (éd.), Understanding Reality Television, Londres/New York, Routledge, 2004, p. 91-110.

3 L'expression «Container » utilisée par Schlingensief fait directement référence à l'émission Big Brother où elle désigne le logement des candidats. Dans les médias, le terme "Container-Show », utilisé pour désigner l'émission, est repris pour l'action de Schlingensief. À la différence du terme "loft » utilisé en France, le terme " container " assimile ses occupants à des objets, voire des marchandises. Dans le changement du titre de l'émission, on constate une certaine prudence de la part du diffuseur français. 
par les spectateurs selon le seul critère de leur sympathie, jusqu'à obtention du gagnant. L'élimination des candidats fut mise en scène comme une reconduction à la frontière. Fidèle à son modèle, ce dispositif donna lieu à trois types de médiations: (1) une retransmission "en direct » (en réalité avec un léger décalage permettant une sélection et un montage des images) sur une webtélé dont le site Internet proposa par ailleurs des informations annexes sur les candidats et le jeu; (2) un rapport quotidien résumant la journée sous la forme d'une narration en épisodes (Schlingensief remplaça l'émission télévisuelle quotidienne par une tribune dans le quotidien allemand Frankfurter Allgemeine Zeitung); (3) une animation de type talk-show sur le lieu du spectacle qui constituait le cadre pour l'annonce des résultats du vote et les éliminations régulières, faisait intervenir un modérateur (Schlingensief ou son double André Wagner) et des experts (dans le cas de Schlingensief des invités du monde politique et culturel) et donnait au public la possibilité de s'exprimer.

La commande avait été passée par Luc Bondy pour le compte du festival international Wiener Festwochen, une vitrine culturelle importante qui bénéficiait à l'époque d'une subvention de l'État autrichien. Or, l'installation-performance fut non seulement conçue, mais aussi annoncée publiquement par Bondy lors de la conférence de presse inaugurale comme une « prise de position politique» (politisches Statement) en réaction directe à la formation du gouvernement d'alliance entre ÖVP et FPÖ début février, qui faisait suite aux élections d'octobre 1999 dans lesquelles le FPÖ était affirmé comme deuxième force politique du pays (derrière le SPÖ et devançant de justesse l'ÖVP) ${ }^{(4)}$. Le spectacle s'inscrit de ce fait directement dans le contexte politique de l'époque qui en fournit la toile de fond, la situation de réception et, comme nous le verrons, le matériau. Dominé sur le plan national par de multiples manifestations d'opposition spontanées dont la créativité fut parfois proche de celle des pratiques situationnistes des années 1960 ou de la révolte étudiante de $1968^{(5)}$, celui-ci fut marqué, sur le plan international, par des réactions diplomatiques virulentes que la réaffirmation solennelle des valeurs fondamentales (notamment le respect des droits de l'Homme et l'intégration européenne), imposée comme préambule au programme du gouvernement par le président autrichien, Klestil, n’avait pas suffi à éviter. Rappelons notamment que les autres États membres de l'Union européenne, sur l'initiative de la France et face à l'impossibilité d'une action de l'Union contre l'un de ses membres, avaient mis en place des sanctions bilatérales, ressenties comme une mise sous tutelle par de nombreux Autrichiens et fortement controversées dans le milieu des spécialistes du droit international. Levées en septembre 2000, elles étaient encore pleinement en vigueur au moment du festival ${ }^{(6)}$. Toutes ces réactions et mesures inhabituelles peuvent être comprises comme des (ré)affirmations identitaires publiques motivées par une " perte d'identité » (Ingeborg Zelinka) collective. Dans le

4 Cette conférence de presse, tout comme la performance dans son ensemble, est documentée dans le film Ausländer raus! Schlingensiefs Container de Paul PoEt, monitorprop entertainment, 2005. Sauf indication contraire, toutes les traductions ont été faites par mes soins.

5 Cf. le chapitre «L'année 2000. La marche vers la crise» et plus particulièrement le sous-chapitre «Protestations et manifestations: un antifascisme modernisé? » dans Patrick Moreau, De Jörg Haider à HeinzChristian Strache. L'extrême droite autrichienne à l'assaut du pouvoir, Paris, Cerf, 2012, p. 284-288.

6 Même la décision de mandater un observatoire des sages pour préparer la sortie de crise ne fut prise que le 30 juin, soit près de quinze jours après la fin de l'installation-performance. 
cadre de l'identité nationale ou européenne reposant autant sur l'affirmation d'un «système social de référence » que sur la « démarcation par rapport à autrui », entendons par là « la crainte que la présence de certains traits communs, de certaines normes et valeurs puisse être perturbée, voire détruite par trop d'altérité » ${ }^{(7)}$.

En 1999/2000, ce phénomène est observable à deux niveaux, formant un effet de cascade. Car le succès électoral du FPÖ, comme celui d'autres partis de la droite populiste en Europe au même moment, reposait bien sûr déjà sur une crise identitaire de ce type par laquelle une partie de la population réagissait aux mutations sociales causées par la mondialisation, l'hétérogénéité culturelle croissante et la nouvelle situation géopolitique issue de l'effondrement du bloc soviétique. Le paysage politique autrichien avait répondu à ces évolutions par un important repositionnement des principaux partis en matière de politique identitaire, ce qui avait probablement favorisé le succès du FPÖ ${ }^{(8)}$. Dans cette " nation tardive au carré » marquée par le " traumatisme de l'exclusion » ( Ausschlusstrauma »), puis celui causé par l'Anschluss ("Anschlusstrauma ») (9), la création d'un «Österreichbewusstsein » dans la fonction d'une identité nationale était encore récente. Comme le souligne Gerald Stieg, cette «'conscience autrichienne', aujourd'hui partagée par une large majorité, n'est pas une donnée naturelle ou historique, mais le résultat d'une 'prise de conscience et d'un processus d'apprentissage' " ${ }^{(10)}$. Porté (après des hésitations du SPÖ) par tous les partis politiques de la Deuxième République à l'exception du FPÖ qui défendait l'idée d'une appartenance des Autrichiens à la nation allemande, celui-ci avait porté ses premiers fruits dans les années soixante et culminé dans les années quatre-vingt-dix. Les Autrichiens estimaient alors à 92-94\% (avec de légères variations selon les années) qu'ils formaient une nation (contre $49 \%$ en 1956 et $70 \%$ en 1964) et se montrèrent plus fiers de leur nation que leurs voisins européens. Inversement, le sentiment d'appartenance à l'Union européenne, à laquelle l'Autriche venait seulement d'adhérer, était encore peu développé et complexifié par le caractère anxiogène de problématiques alors actuelles comme l'élargissement de l'Union à l'Est de l'Europe ou la création de la zone euro. Or si l'ÖVP et le SPÖ, dominants dans la création du "Österreichbewusstsein », avaient adopté après la consolidation de celui-ci

7 Ingeborg Zelinka, "Zur Schaffung von Identität - Österreich und Polen in Europa », in: Karl Аснам, Katharina Scherke (éd.), Kontinuitäten und Brüche in der Mitte Europas. Lebenslagen und Situationsdeutungen in Zentraleuropa um 1900 und um 2000, Vienne, Passagen-Verlag (= Studien zur Moderne 18), 2003, p. 90.

8 Voir Susanne Frölich-Steffen, « Die Identitätspolitik der FPÖ: Vom Deutschnationalismus zum Österreich-Patriotismus », Österreichische Zeitschrift für Politikwissenschaft, n 3 (2004), p. 281-295.

9 Cf. Rudolf Burger, "Die nachträgliche Nation. Gedanken zu einer unvollständigen Gegenwart », Weltstadt Wien. Plattform für urbane An-und Aufregung, conférence du 23.02.2005, http://www.weltstadtwien. org/site/fileadmin/user_upload/Vortrag_Rudolf_Burger.pdf (dernière consultation le 10.09.2013).

10 Gerald Stieg, L’Autriche: une nation chimérique?, Cabris, éditions Sulliver, 2013, p. 131. Sur ce processus, cf. également (y compris pour les résultats d'enquête qui suivent) Ernst BRUCKMÜLLER, "Die Entwicklung des Österreichbewusstseins », in: Robert Kriechbaumer (dir.), Österreichische Nationalgeschichte nach 1945. Die Spiegel der Erinnerung: Die Sicht von innen, Vienne, Böhlau, 1998, p. 369-396. Cf. également Wilhelm KEMPF, « Die Konstruktion nationaler Identität in der Presse seit 45 ", conflict \& communication online, vol. 1, $\mathrm{n}^{\circ} 1$ (2002). Pour une présentation plus complète de l'évolution jusqu'à la fin des années soixante-dix, cf. Félix KreIssler, La prise de conscience de la nation autrichienne: 1938, 1945, 1978, Paris, PUF, 1980. 
une orientation européenne, le FPÖ avait de son côté investi le patriotisme autrichien devenu vacant, revenant par la même occasion également sur sa position initialement pro-européenne pour se positionner comme le défenseur d'une identité nationale menacée. Mais ses principales positions en matière de politique identitaire se trouvaient toujours en contradiction avec l'« Österreichbewusstsein » tel qu'il s'était développé sous la Deuxième République. Celui-ci reposait sur l'idée de l'État-nation ${ }^{(11)}$ et, dans la classification établie par Bernhard Giesen, sur une codification universaliste de la nation (basée sur des valeurs ${ }^{(12)}$. Depuis la fin des années quatre-vingt, il incluait la conscience d'une co-responsabilité du peuple autrichien pour les crimes du national-socialisme (Mitverantwortungsthese) qui avait fini, après l'affaire Waldheim, par remplacer dans la politique mémorielle autrichienne la thèse jusque-là officielle d'un statut de victime de l'Autriche (Opferthese) ${ }^{(13)}$. Le FPÖ, comme le montraient explicitement le programme du parti en 1997 et la campagne électorale ouvertement xénophobe de 1999, continuait à s'appuyer sur une codification primordiale du collectif national (présentant celui-ci comme une entité préexistante à la politique) pour prôner une politique d'immigration qui correspondait, certes, dans les grandes lignes à celle défendue par l'ÖVP, mais qui s'appuyait sur des arguments foncièrement différents ${ }^{(14)}$. S'ajoutait à cela son statut de parti traditionnel qui le distinguait des autres partis nationaux-populistes en Europe et qui avait impliqué depuis ses débuts une continuité personnelle et discursive avec le passé national-socialiste. Aussi, les systèmes sociaux de référence, que ce soit le paysage politique autrichien (depuis 1986), l'internationale libérale ou le groupe libéral au Parlement européen (dans les années quatre-vingt-dix), avaient réagi à ce caractère propre du FPÖ en l'isolant politiquement, établissant ainsi son statut d'altérité au sein du système ${ }^{(15)}$. En février 2000 , si le risque d'un dérapage politique fut mince dans le cadre d'un gouvernement d'alliance fortement dominé par l'ÖVP, l'intégration du FPÖ dans l'exécutif conférait à celui-ci le pouvoir symbolique de représenter l'Autriche (et l'Europe), modifiait de ce fait, tant à l'intérieur qu'à l'extérieur du pays, l'image de la nation sur des points sensibles et finit ainsi par mettre en cause les marqueurs identitaires établis.

C'est sur cette perte d'identité que Luc Bondy mit l'accent lors de la conférence de presse inaugurale de « Bitte liebt Österreich » lorsqu'il motiva l'invitation de Schlingensief par

11 Cf. E. Bruckmüller, « Die Entwicklung des Österreichbewusstseins » (note 10).

12 Pour le rappel de cette classification et son application au spectre politique autrichien, cf. Werner Suppanz, "Antisemitismus und Xenophobie - Konstruktionen des Eigenen und des Fremden in Österreich um 1900 und um 2000 », in: Acham/Scherke (éd.), Kontinuitäten und Brüche in der Mitte Europas (note 7), p. 219-231.

13 Cf. à ce sujet Heidemarie UHL, "Vom Opfermythos zur Mitverantwortungsthese: NS-Herrschaft, Krieg und Holocaust im 'österreichischen Gedächtnis' ", in: Christian Gerbel, Manfred Lechner e.a. (éd.), Transformationen gesellschaftlicher Erinnerung. Studien zur Gedächtnisgeschichte der Zweiten Republik, Vienne, Turia \& Kant, 2005, p. 50-82.

14 Cf. pour l'analyse du programme du parti Werner Suppanz, "Antisemitismus und Xenophobie Konstruktionen des Eigenen und des Fremden in Österreich um 1900 und um 2000 » et au sujet de la campagne électorale la page du Demokratiezentrum Wien où les affiches les plus virulentes sont exposées et commentées, http://www.demokratiezentrum.org/bildstrategien-zusatz/bildstrategienliste.html?index=841 (page consultée le 24.08.2013).

15 Cf. Anton Pelinka, « Die FPÖ in der vergleichenden Parteienforschung. Zur typologischen Einordnung der Freiheitlichen Partei Österreichs ", Österreichische Zeitschrift für Politikwissenschaft, 3 (2002), p. 281-290. 
le «malaise » qu'il ressentait de "vivre dans un pays où le parti conservateur a brisé le tabou en intégrant un parti d'extrême-droite au gouvernement ». Cette justification étonnamment personnelle d'un choix de programmation qui engageait de facto, au-delà de sa personne, le festival dans son ensemble a, me semble-t-il, valeur de programme. La formulation choisie pour désigner l'événement politique incriminé renvoyait nécessairement tant au mythe fondateur antifasciste de l'Union européenne comme système de valeurs qu'au contexte actuel d'une montée des populismes de droite dans plusieurs pays européens. Elle impliquait ainsi une généralisation du propos au-delà de l'actualité autrichienne, une tendance que confirme également la thématique retenue pour le spectacle, la xénophobie, qui faisait explicitement partie de la commande ${ }^{(16)}$ et qui n'était le propre ni du FPÖ ni de l'Autriche. Mais surtout, en concrétisant et en individualisant l'effet produit par cet événement politique, Bondy faisait exister le « malaise ». Aussi, l'installation-performance de Schlingensief n'était pas censée évacuer ce malaise par une affirmation identitaire mais, selon l'expression de Bondy, faire du festival un "podium pour la discussion politique » et offrir au public un « espace permettant de réfléchir à bien des choses » ("Raum geben, über vieles nachzudenken ») ${ }^{(17)}$. Bondy affecta ainsi à l'œuvre d'art non la fonction de joindre sa voix au concert (ou à la cacophonie) des discours identitaires, mais celle de l'interrompre, une fonction qui souligne la singularité de la démarche artistique au sein du contexte décrit plus haut. Selon Hans-Thies Lehmann, elle constitue également l'aspect politique d'une forme de théâtre axée sur la situation théâtrale pour laquelle il venait à l'époque de forger le concept du "postdramatique " ${ }^{(18)}$. Aussi, c'est non par un narratif mais par la structure de son dispositif scénique que Schlingensief répondit à cette commande complexe. Je plaiderai dans la suite de cet article pour une lecture de celui-ci comme emblème interactif.

Cette grille de lecture repose sur la version tripartite de l'emblème telle qu'elle découle de l'Emblematum liber d'André Alciat et devint dominante dans la littérature baroque allemande. Conjuguant image et discours, ce type d'emblème est composé d'un titre (inscriptio), d'une image (pictura) et d'un épigramme (subscriptio) qui explique l'image et lui attribue ainsi une signification. "La double fonction remplie par l'architecture tripartite de l'emblème, la représentation et l'attribution d'une signification, repose sur le fait que l'objet représenté signifie plus qu'il ne représente. La res picta de l'emblème a une capacité indicielle, elle est res significans » ${ }^{(19)}$. Or, à la différence du symbole où ce rapport n'a pas besoin d'être expliqué puisque, selon l'expression de Goethe, l'universel est contenu dans le particulier et peut y être " cueilli », l'emblème repose sur un rapport conventionnel entre image et signification. C'est pour cette raison que plusieurs

16 Bondy rappela ce contrat initial à Schlingensief dans un fax daté du 04.06.2000 reproduit dans Matthias Lilienthal et Claus Philipp (éd.), Schlingensiefs AUSLÄNDER RAUS. BITTE LIEBT ÖSTERREICH, Francfort-sur-le-Main, Suhrkamp, 2000, p. 131.

17 Conférence de presse à l'occasion du lancement de la performance.

18 Voir surtout Hans-Thies Lehmann, «Wie politisch ist postdramatisches Theater? Warum das Politische im Theater nur die Unterbrechung des Politischen sein kann " (2001), in: ID., Das politische Schreiben. Essays zu Theatertexten, Berlin, Theater der Zeit, 2002, p. 11-21.

19 Arthur Henkel et Albrecht Schöne, "Vorbemerkungen der Herausgeber » (1967), in: ID. (éd.), Emblemata. Handbuch zur Sinnbildkunst des XVI. Und XVII. Jahrhunderts, Stuttgart/Weimar, Metzler, 1967/1996, p. XIII. 
significations peuvent être attribuées à une même res picta, comme on le constate notamment au moment de la prolifération des emblèmes à l'époque baroque qui eut pour conséquence la création, autour de nombreuses images, d'un champ entier de significations emblématiques ${ }^{(20)}$. Si les significations potentielles d'une même image sont donc multiples, « dans chaque cas précis, le texte interprétatif de l'emblème établit une relation fixe et univoque entre la res significans et la signification retenue »(21). L'interprétation de « Bitte liebt Österreich » comme emblème repose donc sur la triple hypothèse d'une architecture tripartite de l'action-performance, de son fonctionnement pictural (et non dramatique) et d'une nature conventionnelle du lien existant entre cette image et la signification qui lui est attribuée.

L'inscriptio, visible de loin, est fournie par le slogan «Ausländer raus ». Elle paraphrase le thème imposé, la xénophobie, et devient ainsi le titre de l'emblème, une fonction que lui reconnurent d'ailleurs les deux documentations de l'installationperformance en choisissant ce slogan comme titre ${ }^{(22)}$. Comme dans tout emblème, la pictura provient d'un fond d'images déjà constitué. Elle est reprise sur le mode de la citation. L'image choisie par Schlingensief est de nature composite. Une partie, discursive, est constituée par le décor visuel et sonore issu de la campagne électorale du FPÖ, l'autre partie, narrative, par le dispositif de l'émission télévisée Big Brother. Ces deux parties de l'image fonctionnent comme des icônes de leurs contextes d'origine qu'elles représentent de façon métonymique. Leur utilisation conjointe suggère que ces contextes entretiennent entre eux un rapport d'analogie.

La transformation du narratif de Big Brother en une image supposait de neutraliser le temps transitoire du changement de situation (action) au profit d'une durée (permanence d'un état). Dans "Bitte liebt Österreich », cet effet repose d'une part sur le statut de citation de l'action, d'autre part sur la nature même de cette action, et enfin sur la transplantation du dispositif télévisuel dans l'espace théâtral. Le statut de citation crée une temporalité rétrospective propice à une vue d'ensemble et attribue de surcroît à l'action dans son ensemble le statut d'un signe. Comme cette action (l'élimination progressive des candidats) est directement générée par une règle du jeu qui en fixe à l'avance la durée, le rythme, le déroulement et l'issue (un seul gagnant), son référent reste par ailleurs en dernière instance le jeu lui-même. Le narratif produit par ce jeu, s'il dénote a priori une série de micro-actions aboutissant à un changement de situation, pouvait ainsi être utilisé par Schlingensief comme une brique narrative dont l'ensemble du contenu fut activé dès avant le premier vote du public, au moment de l'annonce publique de la règle du jeu au début du spectacle, et resta intact jusqu'à sa fin. Ce statut particulier de l'action devint évident au moment où des membres du mouvement d'opposition au gouvernement envahirent le site, vandalisèrent l'écriteau et tentèrent de « libérer " les demandeurs d'asile. Au lieu d'accepter cette évolution de la situation et de rebondir sur cet infléchissement imprévu de l'action, Schlingensief se comporta en gardien de l'image et de la règle du jeu et assura par la même occasion la

20 Ibid.

21 Ibid.

22 Lilienthal/Philipp (éd.), Schlingensiefs AUSLÄNDER RAUS. BITTE LIEBT ÖSTERREICH (note 16); P. Poet, Ausländer raus! (note 4). 
conformité de la citation à l'original télévisé: il fit installer un nouvel écriteau et reprit le scénario initialement prévu là où il avait été interrompu.

L'effet statique de l'emblème fut encore renforcé par la dramaturgie du regard qui inverse, dans "Bitte liebt Österreich », les éléments visibles et invisibles de l'émission télévisée Big Brother. Dans Big Brother, le jeu d'élimination et le divertissement télévisuel reposent pareillement sur la (prétendue) visibilité permanente des candidats. La médiation des caméras de surveillance assure la nécessaire transparence du quatrième mur, alors que le montage de ces images aboutissant à une dramatisation sous la forme d'un "real life soap » permet au téléspectateur d'entretenir une relation para-sociale avec les candidats. Par la transplantation du dispositif dans l'espace théâtral, Schlingensief créa la «matérialité » du spectacle (Aufführung ${ }^{(23)}$ ), un espace-temps performatif qui assure pour une durée déterminée la co-présence entre acteurs et spectateurs. Or, comme les images filmées à l'intérieur des " containers » furent pour l'essentiel montrées sur Internet ce qui supposait à l'époque que le spectateur s'absente du lieu théâtral pour les voir ${ }^{(24)}$, l'effet paradoxal de cette co-présence fut que les candidats de «Bitte liebt Österreich » furent dérobés au regard des spectateurs et dissimulés derrière un quatrième mur devenu opaque.

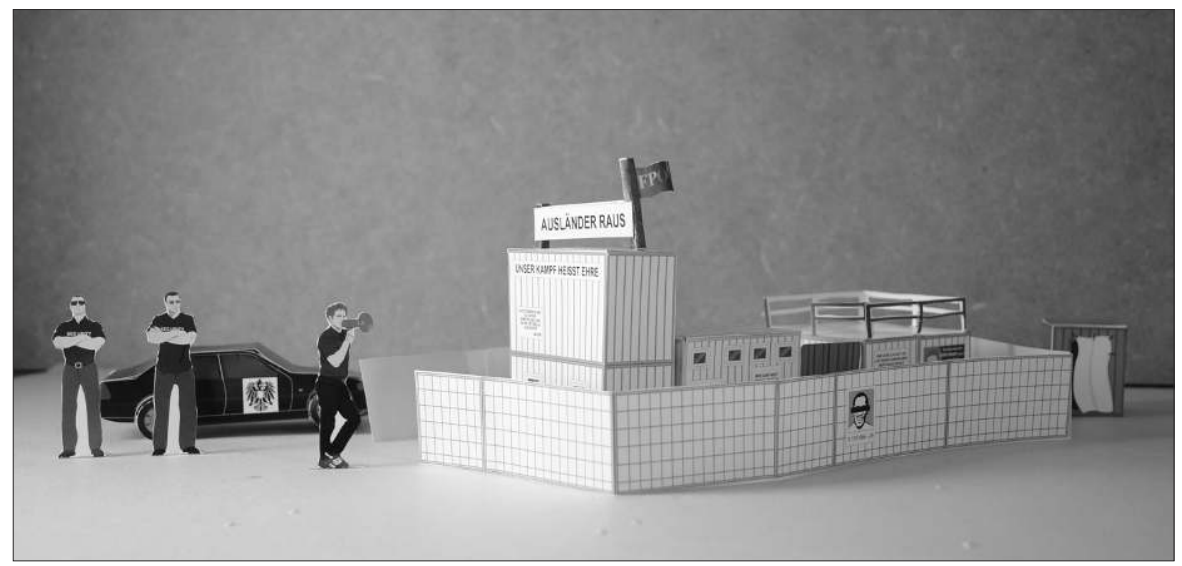

Paul Poet, maquette à monter soi-même, photo Daniel Bascou : village de containers.

Ce qui devint visible dans l'espace théâtral fut en revanche l'envers du décor de l'émission télévisée ${ }^{(25)}$ : un lieu clos placé sous haute protection duquel sortaient une fois par jour deux candidats éliminés. Escortés par le personnel de sécurité et protégés par des barricades anti-émeute, coiffés d'un couvre-chef et dissimulant leur visage

23 Cf. sur ce concept Erika Fischer-Lichte, "Aufführung ", in: ID., Doris Kollesch, Matthias WarsTAт (éd.), Metzler Lexikon Theatertheorie, Stuttgart/Weimar, Metzler, 2005, p. 16-26.

24 Dans l'espace théâtral, seul un petit moniteur à l'extérieur d'un « container » et la "peep-show ", un couloir de palissades, fermé par une grille, qui menait à un endroit d'où on pouvait lorgner dans la cour entre les « containers ", à travers les interstices entre les lattes de la palissade, permirent de voir l'intérieur du dispositif scénique.

25 Une vue extérieure du village de containers ayant accueilli en 2000 les candidats de Big Brother à Hürth près de Cologne est désormais accessible sur Internet : http://www.bb-unzensiert.de/\#!m=10090 (consulté le 15.09.2013). 
derrière un journal, ils n'avaient aucun profil individuel pour les spectateurs. Dans la mesure où ceux-ci n'avaient pas eu l'occasion de construire une relation avec ces candidats dont Schlingensief affirma tantôt qu'ils étaient des acteurs, tantôt qu'ils étaient de vrais demandeurs d'asile, tout en annonçant que leurs CV avaient été modifiés, cette image était purement itérative. Le choix du site au centre historique et touristique de Vienne, le contraste (visuel et conceptuel) avec l'opéra national voisin, l'animation ininterrompue sur et autour des containers et le choix du toit d'un des containers pour les « jeux collectifs» (comme la gymnastique ou les cours d'allemand) eurent pour effet majeur d'exposer, et même d'imposer, le village de containers (invisible pour le téléspectateur) comme objet au regard et de le placer dans un certain nombre de systèmes binaires qui en soulignèrent l'altérité. Au parcours des candidats s'était ainsi substituée dans le regard du spectateur la durée d'une image altérée dont Schlingensief fit le décompte.

Grâce à cette opération d'inversion, les deux parties de la pictura pouvaient entretenir entre elles une relation d'analogie qui reposait simultanément sur l'aspect de l'exclusion et sur celui du jeu. Les deux contextes d'origine se commentent dès lors l'un l'autre. Alors que le discours identitaire du FPÖ peut être lu comme le sous-texte politique de l'émission (que les critiques les plus virulentes de l'émission n'avaient pas manqué d'y déchiffrer ${ }^{(26)}$ ), la brique narrative de Big Brother fonctionne comme la réalisation performative, à travers un jeu purement fictif, des idées populistes et xénophobes du FPÖ dont elle dévoile ainsi la fictionnalité:

"Comme dans Big Brother, il s'agissait de vrais gens dans une vraie situation, mais le cadre dans son ensemble était inventé, voire absurde. On pourrait en tirer une première conclusion: si seul un cadre fictif peut donner réalité à un discours, le caractère fictif de ce discours devient évident ${ }^{(27)}$.

La pictura de l'emblème participe donc à la constitution de significations potentielles liées pour l'essentiel à la construction identitaire par l'exclusion et au populisme, sans fixer une seule signification de façon univoque. De plus, les significations possibles découlent des contextes dont sont issues les images et se réfèrent strictement à ceux-ci. Ils ne contiennent aucune assignation identitaire au sujet de l'Autriche et même le caractère propre du FPÖ, à savoir sa relation particulière au passé national-socialiste, ne fut pas introduit expressément. C’est surtout le discours médiatique et politique déclenché par l'émission Big Brother qui permettait d'inclure cette strate de signification. Celui-ci avait en effet comparé le container de l'émission à un camp de concentration et avait comparé le statut des candidats non seulement à celui de rats de laboratoires, mais également de cobayes humains ${ }^{(28)}$.

26 Cf. Lothar Mikos, Patricia Feise, Katja Herzog e.a., Im Auge der Kamera. Das Fernsehereignis Big Brother, Berlin, Vistas (Beiträge zur Film- und Fernsehwissenschaft, 55), 2000, et D. Biltereyst, "Reality TV, troublesome pictures and panics» (note 2).

27 Mark Siemons, «Der Augenblick, in dem sich das Reale zeigt. Über Selbstprovokation und die Leere », in: Schlingensiefs Ausländer raus (note 16), p. 125-126: "Wie bei Big Brother ging es also um wirkliche Menschen in einer wirklichen Situation, doch der Rahmen als ganzer war völlig erfunden bis zur Absurdität. Daraus ließe sich zunächst die Konsequenz ableiten: Wenn erst ein fiktiver Rahmen einem Diskurs Realität geben kann, erweist sich der Diskurs selbst als fiktiv. »

28 Cf. le chapitre "Der Diskurs um Big Brother" dans Mikos/Feise/Herzog e.a., Im Auge der Kamera (note 26), p. 183-213. 
La fonction proprement interprétative revient ainsi à la subscriptio qui prend dans « Bitte liebt Österreich » une forme orale dans le cadre du "talk-show » autour des containers. Elle est prise en charge dès le début de l'installation-performance par Schlingensief (et son double André Wagner) dans son rôle d'animateur du site. Or, si chacun des énoncés définitoires qui en tiennent lieu établit une signification précise, il est frappant de constater que Schlingensief les multiplia sous la forme de séries de propositions contradictoires dès le début du spectacle: «Das ist Österreich » ( C'est ça, l'Autriche »), «Das ist die FPÖ » ("C'est ça, le FPÖ »), «Das ist die Kronen Zeitung » ( C'est ça, la Kronen Zeitung»), "Das ist Wien » "C'est ça, Vienne »), "Das ist Nazis " ("C’est ça, les nazis »), «das bist du, das bin ich. Das sind Sie " (C'est toi, c'est moi. C’est vous »), etc. La sérialisation des énoncés fit clairement apparaître le caractère conventionnel des attributions de signification dans le cadre de l'emblème et constitua de cette manière une sorte de mode d'emploi de celui-ci. Schlingensief n'en privilégia aucune et évita de se positionner. Interpellant les spectateurs, se contentant souvent de répéter au mégaphone ce que l'un ou l'autre spectateur avait dit, prenant de surcroît différents rôles (dont celui d'un membre du FPÖ), il délégua la constitution du sens très explicitement aux spectateurs. C'est ainsi que l'emblème devint interactif. Comme dans certaines autres performances contemporaines, le dispositif de Schlingensief se concentrait donc avant tout sur la situation théâtrale elle-même et produisait, dans une situation où nul ne peut se tenir en dehors du spectacle, " trois types de spectateurs que l'on peut subdiviser en d'innombrables sous-catégories : celui qui participe au jeu, le voyeur et celui qui refuse de participer. Et aucun de ces rôles ne leur permet de consommer le spectacle à distance, en sécurité et passifs » ${ }^{(29)}$. Le spectateur fut directement impliqué dans le spectacle, il fut rendu conscient de sa co-responsabilité de l'événement et fut amené à faire une expérience du type de celles que Fischer-Lichte décrit, dans le cadre de l'esthéticité du spectacle, comme événement (et non comme mise en scène) ce qui implique un « excès d'attention » de la part du spectateur ainsi que la collision d'oppositions: "Les spectateurs se perçoivent eux-mêmes comme sujets qui influent sur le cours des choses et sont influencés par lui; ils vivent le spectacle comme un processus esthétique et social, voire politique, lors duquel on négocie ses rapports avec les autres, se livre à des luttes de pouvoir, fait et défait des communautés " ${ }^{(30)}$.

Si le quatrième mur opaque ouvrit donc une deuxième scène sur laquelle se déroula le «véritable » spectacle, le contexte politique fournit les conditions pour que l'identité nationale pût en devenir l'objet principal. Là encore, c'est le rapport à l'émission Big Brother qui est déterminant. En se référant au panoptique de Bentham et l'analyse qu'en

29 Jan Deck, «Zur Einleitung: Rollen des Zuschauers im postdramatischen Theater », in: Jan Deck, Angelika Sieburg (éd.), Paradoxien des Zuschauens. Die Rolle des Publikums im zeitgenössischen Theater, Bielefeld, transcript, 2008, p. 15: « drei verschiedene Grundtypen der Zuschauerschaft, die sich in unzählige Untertypen aufteilen können: den Mitspieler, den Voyeur und den Verweigerer. Und keine dieser Rollen ermöglicht ihnen ein geschütztes, passives Konsumieren eines Theaterabends aus sicherer Distanz. »

30 Eriak Fischer-Lichte, "Aufführung ", in: Fischer-Lichte/Kollesch/Warstat (éd.), Metzler Lexikon Theatertheorie (note 23), p. 21-22 : «In ihnen [den Aufführungen, KH] erfahren sich die Beteiligten als Subjekte, die ihren Gang mitbestimmen und sich zugleich von ihm bestimmen lassen. Sie erleben die Aufführung als einen ästhetischen und zugleich sozialen Prozess, in dem die Beziehungen ausgehandelt, Machtkämpfe ausgefochten, Gemeinschaften gebildet und wieder aufgelöst werden. » 
fait Michel Foucault ${ }^{(31)}$, François Jost reconnaît dans le format télévisé (et ses modèles sur Internet ${ }^{(32)}$ ) un "panopticon inversé » ${ }^{(33)}$. Car si le dispositif de Bentham servait à rationaliser les moyens employés pour la surveillance d'une multitude de détenus grâce à une structure circulaire avec une tour de surveillance centrale et des cellules de détenus disposés sur un anneau périphérique, le dispositif de Big Brother inverse en effet le dispositif initial: alors que chez Bentham la périphérie est exposée à la potentialité du regard d'un surveillant dissimulé par la tour qui assure ainsi « le fonctionnement automatique du pouvoir » ${ }^{(34)}$, le format télévisé permet à une multitude de téléspectateurs, invisibles pour les candidats, de les observer à tout moment, mais aussi de s'absenter à leur guise. Jost appelle cela un «'contrat' exhibitionniste » ${ }^{(35)}$. On peut y voir également un phénomène de théâtralité, comme le souligne Jens Roselt:

"Quiconque parle de Big Brother se sert automatiquement des termes clef qui caractérisent la théâtralité: mise en scène, illusion et réalité, authenticité, émotion, voyeurisme, exhibitionnisme et honte. On parle de théâtralité quand il est question de la dialectique du voir et de l'être vu, quand des humains regardent d'autres humains, quand la volonté de voir et la volonté de montrer coïncident - dans une relation réfléchie. Réfléchi veut dire que l'observation n'est pas un effet annexe mais présentée comme condition préalable à la situation observée » ${ }^{(36)}$.

Or, face au « téléspectateur omniscient et omnipotent capable d'éliminer par son vote ceux qui ne lui reviennent pas " ${ }^{(37)}$, les candidats de Big Brother sont dans la situation de devoir construire une image d'eux-mêmes dont ils pensent qu'elle peut leur assurer leur «survie » dans le loft. Ils mettent en place une stratégie de "présentation scénique d'eux-mêmes » ${ }^{(38)}$, ils inventent des personnages et les présentent dans une auto-mise en scène.

Mikos/Feise e.a. relient ce fonctionnement aux mutations de la société décrites plus haut:

«Dans la société différenciée et plurielle, les individus sont soumis à une 'concurrence interindividuelle' où 'chacun doit se présenter comme une personnalité particulière, voire unique' (Winter/Eckert). [...] Les individus n’agissent plus dans le cadre d'identités prédéfinies, mais ils ont la possibilité de choisir parmi plusieurs aspects identitaires qu'ils peuvent avancer comme des pions dans un jeu. Ils doivent faire leurs preuves sur le 'marché identitaire', ils vendent leur

31 Michel Foucault, Surveiller et punir. Naissance de la prison, Paris, Gallimard, 1975, p. 228-264. Jost insiste à juste titre sur le fait que le format télévisuel, à la différence de ses modèles sur Internet, ne produit que l'illusion d'un panoptique inversé dans la mesure où l'instance de médiation manipule l'image. M. Foucault, Surveiller et punir (note 31), p. 234. F. Jost, L'Empire du loft (suite) (note 32), p. 39.

Jens Roselt, «Big Brother: Zur Theatralität eines Fernsehereignisses ", in: Schlingensiefs Ausländer raus (note 16), p. 71: «Wer von Big Brother spricht, bedient sich automatisch der Schlüsselbegriffe, die für Theatralität kennzeichnend sind: Inszenierung, Schein und Sein, Authentizität, Emotion, Voyeurismus, Exhibitionismus und Scham. Von Theatralität spricht man, wenn es um die Dialektik von Sehen und Gesehen-Werden geht, wenn Menschen Menschen betrachten, wenn Schaulust und Zeigelust sich überschneiden - in einem reflektierten Zusammenhang. Reflektiert meint dabei, dass das Moment des Beobachtens nicht bloßer Nebeneffekt ist, sondern als Voraussetzung und Bedingung der betrachteten Situation ausgestellt wird. " 
première et leur deuxième peau (mode et articles de marque) et peuvent se présenter dans une auto-mise en scène singulière. [...] Toutes ces émissions donnent aux individus la possibilité de s'exposer sur le 'marché identitaire' et d'augmenter leur valeur marchande " ${ }^{(39)}$.

La transposition de ce "panoptique inversé » dans l'espace théâtral crée, comme nous l'avons vu, un quatrième mur opaque. Celui-ci a pour conséquence d'inverser une nouvelle fois la répartition entre ombre et lumière. La «tour centrale » dissimule les demandeurs d'asile au point que leur présence - comme celle du surveillant chez Bentham et Foucault - semble pouvoir être suspendue ${ }^{(40)}$. Inversement, les spectateurs se trouvent dans la périphérie visible. Néanmoins, on ne peut pas stipuler que le pouvoir de surveillance soit repassé du côté de la « tour ». La transposition dans l'espace théâtral bloque donc dans un premier temps le fonctionnement du "panoptique inversé ». Parallèlement, le contexte politique de l'époque, l'attention médiatique nationale et internationale dont bénéficie le festival, ainsi que la présence de nombreux touristes sur le lieu choisi pour l'installation-performance créent un second "panoptique inversé » dans lequel le public tient cette fois-ci le rôle des candidats. Schlingensief renforce d'ailleurs cet effet en braquant sur eux une caméra depuis le container et en invitant les touristes à prendre des photos. Les conditions étaient ainsi réunies pour que le public autrichien, par ses réactions à l'emblème interactif, s'expose sur le « marché identitaire » international et joue sa valeur marchande. Autour du container, on pouvait entendre des discussions politiques au sujet de la politique d'immigration, du passé multiculturel du pays, du rapport à l'Allemagne et à l'Europe. Certains spectateurs qui se sentaient visés, de même que la Kronen Zeitung pensèrent pouvoir tirer profit de la situation d'énonciation du spectacle. L'instance d'énonciation étant dans le cas du « Piefke » Schlingensief (et du Suisse Bondy) située à l'extérieur du système social de référence, le spectacle ne pouvait à leurs yeux n'être qu'une assignation identitaire qui, prétendant réaffirmer les repères identitaires du "Österreichbewusstsein », faisait de l'Autriche le pôle d'altérité dans une construction identitaire allemande ou européenne. «Ce n’est pas tous les jours

39 Ibid., p. 53: «In der ausdifferenzierten, pluralisierten Gesellschaft sind die Individuen einer 'interindividuellen Konkurrenz' ausgesetzt, 'in der sie sich als eine besondere, ja einzigartige Persönlichkeit darstellen müssen’ (Winter/Eckert). [...] Die Individuen handeln nicht mehr zwanghaft im Rahmen festgefügter Identitäten, sondern haben die Wahl zwischen verschiedenen Identitätsaspekten, die sie spielerisch einsetzen können. Sie müssen sich auf dem 'Identitätsmarkt' bewähren, sie tragen ihre erste und zweite Haut (Mode und Markenartikel) zu Markte und können sich in einzigartiger Selbstinszenierung darstellen. [...] All diese Sendungen bieten den Individuen die Möglichkeit, sich auf dem 'Identitätsmarkt' darzustellen und ihren Marktwert zu erhöhen. »

40 C'est là l'avis du journaliste Thomas Rottenberg dans son commentaire télévisé sur place: «J'ai appris que les vrais événements ici ont lieu dans la tête. Il y a des gens dans le container, à la manière de l'émission Big Brother, et en réalité, ils ne font rien. Et puis, il y a des gens devant qui réfléchissent à ce que ces gens qui ne font rien pourraient bien faire. Et tout ce qui se passe se passe dans la tête des gens. Des préjugés sont projetés contre ce container. Et ce qui se passe à l'intérieur? Le container pourrait tout aussi bien être vide. Cela ne fait aucune différence " / "Ich habe gelernt, dass die eigentlichen Ereignisse hier im Kopf passieren. Da sitzen Menschen drinnen im Container herum, so ähnlich wie bei Big Brother auch, und tun eigentlich nichts. Und dann stehen Leute davor und denken darüber nach, was die nichts Tuenden eigentlich tun könnten. Und alles, was hier passiert, passiert in den Köpfen der Leute. Es werden einfach Voruteile an diese Container-Wand geknallt. Und was da drinnen passiert? Der Container könnte genauso gut leer sein. Es ist völlig egal » ("Zeit im Bild 3, ORF, 13.06.2000 », in: Schlingensiefs Ausländer raus [note 16], p. 98). 
qu'on obtient à si peu de frais un profilage éthique en même temps qu'une clarification des relations hégémoniques en Europe » ${ }^{(41)}$, avait déjà ironisé Rudolf Burger au sujet des sanctions bilatérales européennes. Mais Schlingensief, loin d'être victime de ce type de contradictions, les pointait lui-même. Les réactions politiques et diplomatiques furent à leur tour constamment mises à distance, par exemple lorsque Schlingensief nomma parmi les spectateurs un « représentant de la résistance [sic!]» («Widerstandsbeauftragter »), avant d'ajouter: «Ce sont là les images que l'Europe adore ». L'emblème interactif faisait ainsi apparaître dans le double panoptique le caractère performatif de l'identité nationale ou européenne, il exhiba les mécanismes de construction de cette identité et accorda une place bien plus importante au malaise identitaire que ne veut l'admettre sa lecture dramatique. Parmi les moments les plus intéressants de la semaine figurent d'ailleurs ceux où le piège de l'ambivalence se refermait sur ceux des spectateurs qui croyaient pouvoir applaudir le «message » du spectacle : ainsi lors des applaudissements au moment du dévoilement de l'écriteau «Ausländer raus» ou encore au moment des expulsions. Ces moments mettaient à nu le mécanisme d'une construction identitaire basée sur l'exclusion chez ceux-là même qui croyaient le combattre.

Certains spectateurs venaient très régulièrement sur le site et tenaient de véritables rôles, parfois même en " costume » et avec des accessoires (drapeau, décorations, écriteau " Je suis Autrichien et ai honte de cette action ») au point que Paul Poet, le réalisateur du site Internet de «Bitte liebt Österreich » et du film documentaire Ausländer raus. Schlingensiefs Container, décida de les inclure dans la pictura, en leur consacrant des figurines nommés «Autrichien » dans la maquette du lieu scénique vendue avec le DVD.

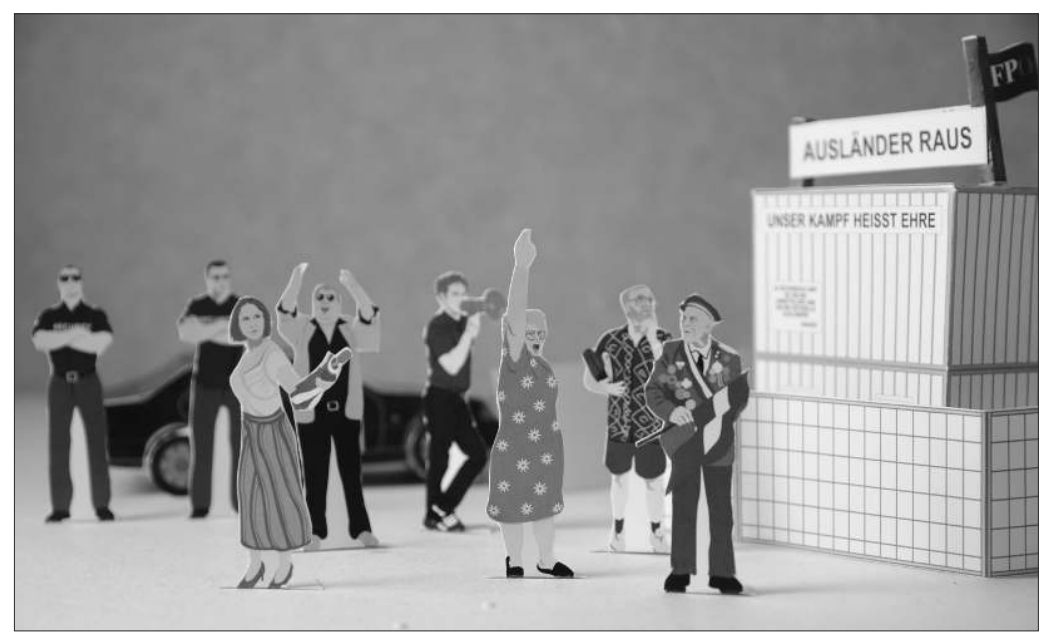

Paul Poet, maquette à monter soi-même, photo Daniel Bascou : personnages dénommés « Autrichiens".

Il est en effet tentant de supposer que, tout comme le panoptique inversé, l'emblème interactif dispose d'une structure à deux échelles. Basant son analyse essentiellement sur le

41 Rudolf Burger, "Austromanie oder der antifaschistische Karneval », Merkur. Deutsche Zeitschrift für europäisches Denken, vol. 54, $\mathrm{n}^{\circ} 5$ (2000), p. 392: «Derart billig kommt man so bald nicht wieder zu einer ethischen Kontur und zu einer Klarstellung der hegemonialen Verhältnisse in Europa. » 
film de Poet, Denise Varney cherche à faire de cette seconde scène le lieu d'une représentation narrative de l'Autriche sous la forme d'un Lehrstück à la dialectique négative:

«Si on prend les incidents et événements intervenus pendant la semaine comme un ensemble, il est possible de percevoir, tout comme dans un scenario inventé ou une fable, un narratif qui concerne un peuple suspicieux et angoissé qui, sous l'influence du malveillant Haider, éjectait des marginaux et des étrangers de sa communauté. Représenté avec le public comme participants, ce scénario négatif présentait une situation déplorable qui, de peur que le pays succombe (une nouvelle fois) à des forces malveillantes, poussait à agir » ${ }^{(42)}$.

Mais comme le suggère déjà le découpage du film en "jours », autrement dit en épisodes narratifs, celui-ci propose une dramatisation à partir d'un montage. Tout comme les rapports que Schlingensief publia quotidiennement dans la Frankfurter Allgemeine Zeitung, le film reproduit ainsi fidèlement le type de médiatisation que propose dans Big Brother l'émission quotidienne présentant le résumé de la journée sous la forme d'un « real life soap » et dont François Jost souligne l'importance:

«Loft Story est à la rencontre de l'aspiration toujours plus grande de l'individu à se penser comme l'acteur de son quotidien et du téléspectateur, qui veut non seulement jouir de sa position d'observateur, mais retrouver dans la vie des autres les schémas narratifs qui l'émeuvent ordinairement dans la fiction. Loin d'être une fenêtre sur la réalité, ce programme a eu l'habileté d'emprunter à l'image que la télévision en donne à travers ses fictions et ses reportages ${ }^{(43)}$.

En forçant le trait par un générique répété qui souligne cette structure en épisodes, ainsi qu'une dramatisation outrancière du danger du « retour du passé » au début du film, Poet pousse à mon sens le spectateur à mettre ce format à distance. Le " real life soap " de l'Autriche fait partie de l'emblème, il expose les différents "rôles " disponibles dans le commerce identitaire. Dans sa maquette, Paul Poet propose comme personnages, à côté des "Autrichiens " et des "étrangers ",

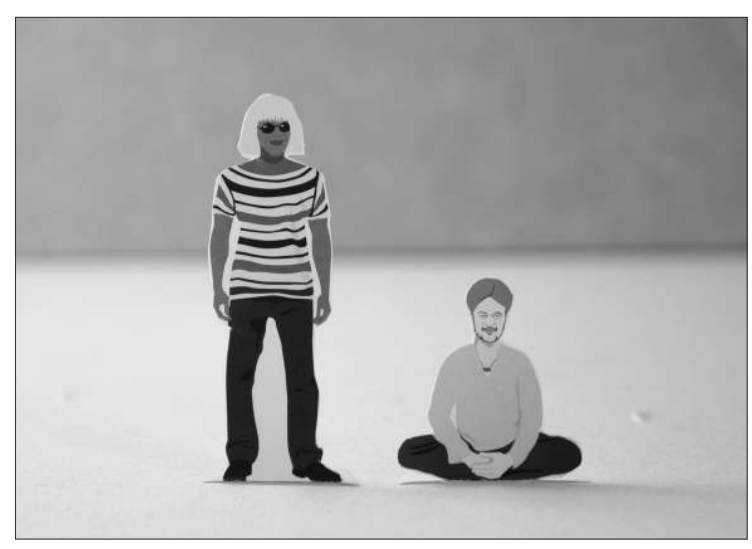

Paul Poet, maquette à monter soi-même, photo Daniel Bascou : personnages dénommés « étrangers ».

42 Denise VArney, «'Right now Austria looks ridiculous': Please Love Austria! - Reforging the Interaction between Art and Politics ", in: Tara Forrest, Anna Teresa Scheer (éd.), Christoph Schlingensief. Art without borders, Bristol, UK / Chicago, USA, 2010, p. 107-108: "Taking the incidents and events that transpired during the week as a whole, it is possible to perceive a narrative, as in an imagined scenario or fable, that concerned a suspicious and fearful people who, under the influence of the malevolent Haider, evicted outsiders and foreigners from their community. Played out with the public as participants, this negative scenario presented a deplorable situation that demands action, lest the country succumb (once again) to malevolent forces. »

43 F. Jost, L'Empire du loft (la suite) (note 32), p. 118. 
l'" activiste ", le « manifestant " et Schlingensief lui-même. L'emblème, image signifiante (Sinnbild), est une image qui pousse à réfléchir (Denkbild), une réalité dont tiennent compte Matthias Lilienthal et Claus Philipp dans leur documentation du projet: leur livre se présente sous la forme d'un recueil de documents et de textes critiques. Il renonce entièrement à une forme narrative.

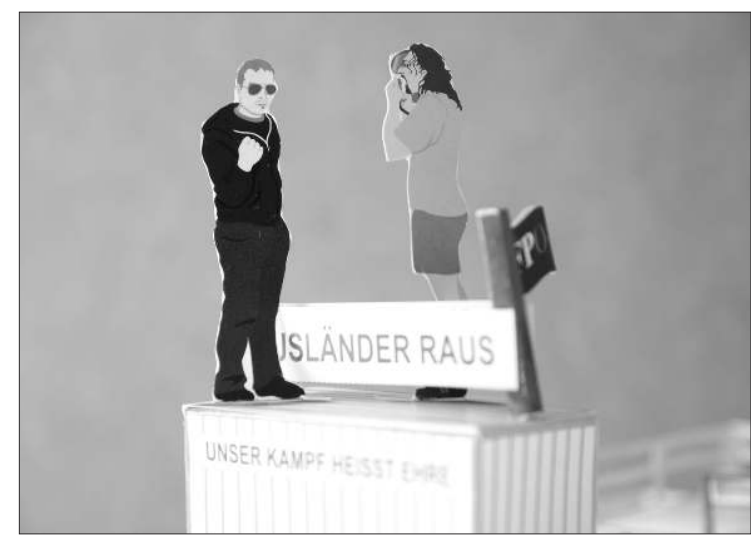

Paul Poet, maquette à monter soi-même, photo Daniel Bascou : personnages dénommés " activiste » et « manifestant».

\title{
Zusammenfassung
}

Im Jahr 2000 hatte die Integration der FPÖ in die österreichische Regierung zu einem nationalen und europäischen "Identitätsverlust“ geführt, auf den der damalige künstlerische Leiter der Wiener Festwochen, Luc Bondy, mit der Einladung Schlingensiefs als politisches Statement reagierte. Dennoch steht die Aktion „Bitte liebt Österreich“ nicht auf derselben Ebene wie diejenigen der Oppositionsbewegung in der österreichischen Zivilgesellschaft. Die Übernahme des TV-Formats Big Brother, das hier als umgekehrtes Panoptikum analysiert wird, sowie die profunde Veränderung, die es beim Transfer in den theatralischen Raum erfährt, erlaubten es Schlingensief, ein potenziertes Emblem $z$ u schaffen. Statt selbst eine Identitätszuweisung zu unternehmen, wurde die sinngebende subscriptio des Emblems den Zuschauern überantwortet, die sich dadurch auf dem internationalen "Identitätsmarkt" profilieren mussten. Sieht man das Publikum als Teil der Aktion und damit des Emblems, erscheint der Identitätsmarkt selbst als das eigentliche Thema von Schlingensiefs Aktion.

\begin{abstract}
In 2000, the integration of the FPÖ in the Austrian government led to a "loss of identity" in Austria and Europe. Luc Bondy, the artistic director of the festival Wiener Festwochen, reacted by inviting Schlingensief; this was meant to be a political statement. However, the performance "Bitte liebt Österreich" did not unfold on the same level as the actions of the opposition movement within civil society. By taking up the setting of the reality-show Big Brother, which shall be interpreted in this article as an "inversed panopticon", and by modifying it in some crucial points like putting it into the theatrical space, Schlingensief created a powerful emblem. Instead of defining Austrian identity, he left the emblem's subscriptio to the spectators, who were thus forced to create their role on the "identity-market". If the public is seen as a being part of the play-and of the emblem - this "identity-market" then turns out to be the real subject of Schlingensief's performance.
\end{abstract}

